\title{
New Onset Vertigo After COVID-19 Infection. A Case Report
}

\author{
Karam R. Motawea $^{1}$ (i) $\cdot$ Fatma A. Monib $^{2}$
}

Received: 1 June 2021 / Accepted: 27 June 2021 / Published online: 4 July 2021

(C) Association of Otolaryngologists of India 2021

\begin{abstract}
In addition to the known pathophysiological sequelae of the SARS-COV-2 virus and its related respiratory symptoms, several studies have recently reported cardiovascular, gastrointestinal, and neurological symptoms of new-onset after a history of infection. Vertigo is a symptom indicating dysfunction of the vestibular system and this report of a 60 -year-old female patient points out the possible association between new-onset vertigo and SARS-COV-2 infection. A 60-year-old diabetic and hypertensive female patient with a 9-days history of COVID-19 symptoms presented to the emergency department with acute vertigo attack without nausea or vomiting. A full physical examination showed no other auditory or neurological symptoms and she never experienced vertigo before. Laboratory findings confirmed a current COVID-19 infection. The patient was managed with antihypertensive, anti-vertigo medications, and COVID-19 protocol for mild cases. After discharge, the patient was advised to continue the anti-vertigo drugs and COVID-19 medications. SARSCOV-2 may lead to vestibular neuritis causing vertigo and other related symptoms, but more well-designed observational studies with a large sample size are needed to establish a definite association between COVID-19 and vertigo.
\end{abstract}

Keywords COVID-19 - Vertigo · Vestibular neuritis · SARS-COV-2

Karam R. Motawea

karammetawea450@gmail.com

1 Faculty of Medicine, Alexandria University, Alexandria, Egypt

2 Faculty of Medicine, Assiut University, Assiut, Egypt

\section{Introduction}

The ongoing SARS-COV-2 pandemic has resulted in millions of severe cases and deaths all over the world. COVID-19 can lead to severe complications during and after recovery from the infection as cardiovascular and gastrointestinal disorders [1, 2]. Some studies reported post-covid neurological complications [3]. Vertigo is a type of dizziness, defined as a false impression of movement brought about by lesions in the central or peripheral vestibular system [4]. The association between SARSCOV-2 and ear disorders has not been confirmed yet. However, some studies reported an association between SARS-COV-2 infection and newly diagnosed vestibular neuritis $[5,6]$. Those patients developed acute vertigo attacks after the infection that lasted for variable periods after recovery from COVID-19 [6]. Here, we report a case of a 60-year-old female patient who did not suffer from any ear disease before, with new-onset vertigo after SARSCOV-2 infection.

\section{Case Presentation}

A 60-year- old female patient with a 9 days history of fever, cough, hoarseness of voice, sore throat, runny nose, dyspnea, myalgia, and fatigue, presented to the emergency department with acute vertigo attack without nausea or vomiting. Her blood pressure was $180 / 100 \mathrm{~mm} / \mathrm{Hg}$. She was given an antihypertensive agent to lower her blood pressure and intravenous access was done with anti-vertigo drugs to control the vertigo attack. Cranial nerves and other neurological examinations were normal without nystagmus or auditory disorders. The physician prescribed her betahistine dihydrochloride $24 \mathrm{mg}$ daily as anti-vertigo medication. Based on the previous contact with a confirmed 
Table 1 Patient Laboratory results

\begin{tabular}{lll}
\hline Parameter & Result & Normal range \\
\hline WBCs & 9600 & $4000-11,000$ \\
Neutrophils & $64 \%$ & $40-70$ \\
Lymphocytes & $28 \%$ & $20-45$ \\
ESR 1st hour & $31 \mathrm{~mm} / \mathrm{hr}$ & Up to 10.0 \\
ESR 2nd hour & $64 \mathrm{~mm} / \mathrm{hr}$ & Up to 20.0 \\
C-RP & Positive $(7.9) \mathrm{mg} / \mathrm{dl}$ & Negative: less than 6.0 \\
PH & 7.466 & $(7.350-7.450)$ \\
PO2 & $77 \mathrm{mmHg}$ & $(80-105)$ \\
PCo2 & $30.8 \mathrm{mmHg}$ & $(35-45)$ \\
SPo2 & $90 \%$ & $(95-100)$ \\
\hline
\end{tabular}

COVID-19 patient who lives with her at the same home and her current symptoms, laboratory investigations were done and elucidated that she was infected with SARSCOV-2. Other laboratory results are shown in Table 1. The physician prescribed her non-specific COVID-19 medications and advised her to isolate herself at home. The patient continued on betahistine dihydrochloride $24 \mathrm{mg}$ daily with COVID-19 medications. She has a medical history of diabetes and hypertension and surgical history of appendectomy, vertebral fixation, and splenectomy. She never had a vertigo attack or any ear disease before. The patient was followed up after recovery from COVID-19 for one month and a half and still suffers from vertigo but to a lesser extent as the patient continued on anti-vertigo medication. The physician told the patient to continue on betahistine hydrochloride $24 \mathrm{mg}$ daily for three months.

\section{Discussion}

We introduce a case of 60 -year- old female patient with new-onset vertigo after COVID-19 infection. The patient did not suffer from nausea, vomiting, nystagmus, auditory, or any other neurological disorders excluding central nervous system involvement. Central vestibular lesions cause nausea, vomiting, vertigo, and other neurological disorders [4]. The patient has a negative medical history of vertigo and any other ear disease. Vertigo persisted for the one month and a half follow-up after recovery from the infection.

Some studies reported the role of some viral infectons in vertigo pathogenesis as Epstein-Barr virus, cytomegalovirus, rubella virus, adenoviruses, influenza virus $A$, influenza virus B, and herpes simplex virus [7]. Direct vestibular nerve infection by the virus is a possible mechanism as proposed by some studies [8]. Milionis et.al linked vestibular neuritis to the increase in levels of Plasma fibrinogen and C-reactive protein (CRP) as a result of the inflammatory event of the viral infection [9]. Bumm et al. supported the immunological theory in vestibular neuritis pathogenesis. They detected the presence of T4 T-helper and $\mathrm{T} 8 \mathrm{~T}$-suppressor cells by specific monoclonal antibodies in inner ear diseases (e.g., vestibular neuritis, sudden hearing loss, Bell's Palsy, and Menière's disease) [10]. Moreover, a novel and interesting link between COVID-19 and newly diagnosed hearing loss, vertigo, otalgia, and tinnitus have been reported [11]. Some reports linked vertigo to post-covid infection $[5,6]$. This association is not elucidated sufficiently and the published studies about it are few. The mechanism of vestibular neuritis in SARSCOV-2 is probably by viral or post-viral inflammatory disorder [12]. The best treatment modality in these patients is symptomatic as antihistaminic, anti-emetics, benzodiazepines, and anticholinergics, and intravenous access is generally done in the initial stages, as the oral route of administration in these patients is poor [13]. In severe cases, corticosteroids could also be used [14].

In conclusion, vertigo as a result of vestibular neuritis may occur as a complication of SARS-COV-2 infection. More research is required to confirm if there is a real association between new-onset vertigo and COVID-19.

Funding All the authors declare that they didn't receive any fund source for the preparation of this article.

\section{Declaration}

Conflict of Interest The authors declare no conflict of interest.

\section{References}

1. Madjid M, Safavi-Naeini P, Solomon SD, Vardeny O (2020) Potential Effects of Coronaviruses on the Cardiovascular System: A Review. JAMA cardiology 5(7):831-840

2. Tian Y, Rong L, Nian W, He Y (2020) Review article: gastrointestinal features in COVID-19 and the possibility of faecal transmission. Aliment Pharmacol Ther 51(9):843-851

3. Whittaker A, Anson M, Harky A (2020) Neurological Manifestations of COVID-19: a systematic review and current update. Acta Neurol Scand 142(1):14-22

4. Karatas M (2008) Central vertigo and dizziness: epidemiology, differential diagnosis, and common causes. Neurologist 14(6):355-364

5. Malayala SV, Raza A (2012) A case of COVID-19-induced vestibular neuritis. Cureus 12(6):e8918

6. Vanaparthy R, Malayala SV, Balla M (2012) COVID-19-induced vestibular neuritis, hemi-facial spasms and Raynaud's phenomenon: a case report. Cureus. 12(11):e11752

7. Hirata T, Sekitani T, Okinaka Y, Matsuda Y (1989) Serovirological study of vestibular neuronitis. Acta Otolaryngol Suppl 468:371-373 
8. Greco A, Macri GF, Gallo A, Fusconi M, De Virgilio A, Pagliuca $\mathrm{G}$ et al (2014) Is vestibular neuritis an immune related vestibular neuropathy inducing vertigo? J Immunol Res 2014:459048

9. Milionis HJ, Mittari V, Exarchakos G, Kalaitzidis R, Skevas AT, Elisaf MS (2003) Lipoprotein (a) and acute-phase response in patients with vestibular neuronitis. Eur J Clin Investig 33(12):1045-1050

10. Bumm P, Schlimok G (1986) Lymphocyte subpopulations and HLA-DR determinations in diseases of the inner ear and Bell's palsy. HNO 34(12):525-527

11. Maharaj S, Bello Alvarez M, Mungul S, Hari K (2020) Otologic dysfunction in patients with COVID-19: a systematic review. Laryngoscope Investig Otolaryngol 5(6):1192-1196
12. Lee H, Sohn SI, Cho YW, Lee SR, Ahn BH, Park BR et al (2006) Cerebellar infarction presenting isolated vertigo: frequency and vascular topographical patterns. Neurology 67(7):1178-1183

13. Norrving B, Magnusson M, Holtås S (1995) Isolated acute vertigo in the elderly; vestibular or vascular disease? Acta Neurol Scand 91(1):43-48

14. Strupp M, Zingler VC, Arbusow V, Niklas D, Maag KP, Dieterich $\mathrm{M}$ et al (2004) Methylprednisolone, valacyclovir, or the combination for vestibular neuritis. $\mathrm{N}$ Engl $\mathrm{J}$ Med 351(4):354-361

Publisher's Note Springer Nature remains neutral with regard to jurisdictional claims in published maps and institutional affiliations. 